\title{
Intérêt de la Marge de Manœuvre Situationnelle pour le ciblage des situations à risque de Troubles Musculo-Squelettiques
}

Étude de cas dans une industrie de la métallurgie

The advantage of situational operational leeway in the identification of

Musculoskeletal Disorders risk situations

Maxime Norval, Mohsen Zare, René Brunet, Fabien Coutarel et Yves Roquelaure

\section{(2) OpenEdition}

Édition électronique

URL : http://journals.openedition.org/activites/4588

DOI : 10.4000/activites.4588

ISSN : $1765-2723$

Éditeur

ARPACT - Association Recherches et Pratiques sur les ACTivités

Référence électronique

Maxime Norval, Mohsen Zare, René Brunet, Fabien Coutarel et Yves Roquelaure, « Intérêt de la Marge de Manœuvre Situationnelle pour le ciblage des situations à risque de Troubles

Musculo-Squelettiques ", Activités [En ligne], 16-2 | 2019, mis en ligne le 15 octobre 2019, consulté le 17 octobre 2019. URL : http://journals.openedition.org/activites/4588 ; DOI : 10.4000/activites.4588

Ce document a été généré automatiquement le 17 octobre 2019.

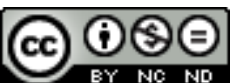

Activités est mis à disposition selon les termes de la licence Creative Commons Attribution - Pas d'Utilisation Commerciale - Pas de Modification 4.0 International. 


\section{Intérêt de la Marge de Manœuvre Situationnelle pour le ciblage des situations à risque de Troubles Musculo-Squelettiques}

Étude de cas dans une industrie de la métallurgie

The advantage of situational operational leeway in the identification of

Musculoskeletal Disorders risk situations

Maxime Norval, Mohsen Zare, René Brunet, Fabien Coutarel et Yves

Roquelaure

\section{NOTE DE L'ÉDITEUR}

Article soumis le $1^{\mathrm{er}}$ juin 2018, accepté le 22 mars 2019

\section{Introduction}

Les Troubles Musculo-Squelettiques (TMS) représentent la première cause de maladie professionnelle dans les pays industrialisés (Roquelaure, 2015 ; Roquelaure, Ha, Leclerc, Touranchet, Sauteron, \& Melchior, 2006 ; Roquelaure, Petit, Meyer, Leclerc, \& Mairiaux, 2013). En constante évolution dans ces pays depuis les années 1990, les TMS représentent un phénomène épidémique qui prend sa source dans l'intensification du travail caractérisée par la généralisation des nouvelles méthodes de production et d'organisation du travail dans les industries (Caroly, Simonet, \& Vézina, 2015; Davezies, 2007 ; Gollac \& Volkoff, 1996 ; Parot-Schinkel, Descatha, Ha, Petit, Leclerc, \& Roquelaure, 2012 ; Roquelaure, Leclerc, Coutarel, Brunet, Caroly, \& François, 2012 ; Westgaard \& Winkel, 2011). Les TMS ont des effets importants sur la santé des individus, mais aussi sur le rendement des entreprises (Ringelberg \& Koukoulaki, 2002 ; 
Roquelaure etal., 2012). Les démarches de prévention des risques de TMS sont structurées pour permettre l'amélioration des situations de travail (Guérin, Laville, Daniellou, Duraffourg \& Kerguelen, 1997 ; St-Vincent, Vézina, Bellemare, Denis, Ledoux, \& Imbeau, 2014). Dans la plupart des modèles de prévention, l'évaluation du risque est la première étape, menée par les préventeurs en entreprises, pour rechercher les situations de travail les plus critiques dans l'apparition de TMS. Cette phase initiale permet aux préventeurs d'estimer le risque d'apparition de la pathologie et d'engager des interventions pour transformer les situations les plus critiques. Cette phase initiale d'évaluation du risque est considérée comme une aide aux choix stratégiques de prévention. Les préventeurs en entreprises ne pouvant pas réaliser des études détaillées sur tous les postes, par manque de ressources et/ou de compétences en interne, utilisent classiquement "des outils simples" d'évaluation du risque pour sélectionner les postes les plus problématiques (David, 2005 ; Falzon, 2013 ; Malchaire, Gauthy, Piette, \& Strambi, 2011; Takala, Pehkonen, Forsman, Hansson, Neumann, \& Westgaard, 2010).

2 La définition des « outils simples » est donnée par Aptel et ses collègues (2000, p. 7): «Les outils simples sont des outils d'évaluation des facteurs de TMS qui ne nécessitent pas d'appareil de mesure particulier. Ils sont utilisables directement sur le terrain et se basent sur l'observation directe de l'activité de travail du salarié ou sur l'enregistrement vidéo de celle-ci et/ou sur l'échange avec le travailleur ». Les outils simples d'observation type check-list, les questionnaires et auto-questionnaires, et les outils d'évaluation créés en interne aux grandes entreprises sont fréquemment utilisés par les préventeurs (David, 2005; Malchaire et al., 2011; Ringelberg \& Koukoulaki, 2002 ; Takala et al., 2010 ; Zare, 2015). Plusieurs revues d'outils simples ont étudié les limites et les avantages de ces outils, détaillés ci-après (Barrero, Katz, \& Dennerlein, 2009 ; Stock, Fernandes, Delisle, \& Vézina, 2005 ; Zare, Biau, Brunet, \& Roquelaure, 2017). Les approches dominantes classiques de l'évaluation du risque TMS, avec des outils simples, se concentrent sur l'identification des expositions, essentiellement en lien avec la biomécanique du mouvement, qui souligne principalement les conditions pathogènes de sollicitation en termes d'intensité, de posture, de durée et de fréquence (Coutarel, Caroly, Vézina, \& Daniellou, 2015 ; Coutarel \& Petit, 2013 ; Guérin et al., 1997 ; St-Vincent et al., 2014).

3 La fiabilité et la répétabilité des résultats de ces outils simples sont critiquées notamment par rapport à la divergence des critères intrinsèques aux outils simples (p. ex. précision des données recueillies, modèle étiologique des TMS de référence, échelle d'interprétation, zones corporelles ciblées, nombre et type d'informations prises en compte) et la diversité de leurs critères d'usage (p. ex. expérience utilisateur, connaissance de l'activité, focale d'observation choisie, méthode de hiérarchisation des résultats; Bao, Howard, Spielholz, silverstein, \& Polissar, 2009; Chiasson, Imbeau, Aubry, \& Delisle, 2012 ; Ringelberg \& Koukoulaki, 2002 ; Roquelaure et al., 2013 ; Zare, Biau, Brunet, Gourlay, \& Roquelaure, 2015). Le manque de fiabilité des résultats va augmenter avec les instabilités inhérentes de l'activité, comme les variabilités liées aux conditions de réalisation (technico-organisationnelles et diversification des produits), et liées aux individus (inter-intra individuelles et diversité des stratégies de réalisation; Badets, Merlo, \& Pilniere, 2017; Coutarel et al., 2015 ; Gaudez, Gilles, \& Savin, 2016 ; Guérin, et al., 1997 ; Koukoulaki, 2014 ; Mathiassen, 2006 ; Mathiassen, Liv, \& Wahlström, 2012 ; Zare et al., 2015). En plus, des instabilités décrites, on observe un 
enrichissement des connaissances scientifiques sur le modèle étiologique des TMS aboutissant aujourd'hui à un consensus autour de la multi-causalité (multi-factorialité) des facteurs de risque (Armstrong, Buckle, Lawrence, Hagberg, Jonsson, \& Kilbom, 1993 ; Bodin, Garlantézec, Costet, Descatha, Fouquet, Caroly, \& Roquelaure, 2017 ; Bourgeois \& Hubault, 2005; Hagberg, Kuorinka, \& Forcier, 1995; Karsh, 2006; Roquelaure, 2015, 2018 ; Widanarko, Legg, Devereux, \& Stevenson, 2014). La multicausalité des TMS et les variabilités constatées dans l'activité de travail questionnent la pertinence des outils simples d'évaluation et leurs intérêts dans la démarche de prévention des TMS (Coutarel et al., 2015 ; Coutarel, Daniellou, \& Dugué, 2003 ; Leplat, 2006). Ces constats créent par exemple, pour les utilisateurs des outils simples, des difficultés de représentativité des résultats lors de la recherche des situations critiques (situations les plus à risque), et ne facilitent donc pas, non plus, le suivi des effets des projets de transformation sur les expositions (Bourgeois, \& Hubault, 2005 ; Bouville, 2013 ; Roquelaure et al., 2012 ; Zare, 2015).

Dans le champ de l'intervention ergonomique, entendue comme "intervention complexe » (Albert, Vézina, Bilodeau, \& Coutarel, 2017 ; Coutarel et al., 2005 ; Guérin, et al., 1997), l'histoire et les nouvelles connaissances conceptuelles en ergonomie de l'activité ont mis l'accent, dans une approche systémique et développementale, sur les contraintes étudiées, mais aussi sur les ressources de la situation de travail comme pouvant permettre la régulation de l'activité (malgré et avec les contraintes individuelles, environnementales et organisationnelles ; St-Vincent et al., 2014 ; Vézina, 2001). Plusieurs publications scientifiques en ergonomie de l'activité et en prévention des TMS invitent à un renouvellement de l'approche de la pathologie par la prise en compte du principe de régulation de l'activité et de la marge de manœuvre en situations de travail (Caroly et al., 2015; Coutarel et al., 2015 ; Cuny-Guerrier, 2017; Guérin, et al., 1997 ; St-Vincent et al., 2014 ; Vézina, 2001). L'activité des opérateurs apparait alors, non pas comme une simple application de consignes prescrites, mais comme un processus de régulation visant à favoriser l'atteinte des objectifs (personnels et fixés par l'entreprise), en gérant les variations du système technico-organisationnel et ses propres variabilités individuelles (Noulin, 2002; St-Vincent et al., 2014). Le processus de régulation a pour objectif, plus ou moins conscient, la conservation de l'équilibre entre le maintien de la santé et l'atteinte des objectifs correspondant aux tâches (St-Vincent et al., 2014). Les possibilités de régulation de l'activité augmentent lorsque la marge de manœuvre des opérateurs en situation de travail augmente également (Coutarel et al., 2015 ; Coutarel et al., 2003). La marge de manœuvre est issue de la rencontre entre les caractéristiques d'un milieu professionnel et celles du ou des travailleurs concernés (Coutarel, 2004 ; Coutarel \& Petit, 2013 ; Durand, Vézina, Baril, Loisel, Richard, \& Ngomo, 2008). Cette rencontre est situationnelle d'après Coutarel et ses collègues $(2015$, p. 15$)$ :

«La marge de manœuvre situationnelle (MMS) constitue la possibilité pour l'opérateur, dans une situation précise, d'élaborer un mode opératoire (ou une stratégie de réalisation) efficient (c'est-à-dire efficace pour la performance et compatible avec la préservation de soi, voire le développement de soi par le travail)».

Il existe plusieurs niveaux de MMS avec des effets différents : si la marge de manœuvre est importante (suffisance MMS), plusieurs stratégies de réalisation sont possibles, l'opérateur peut alterner et en inventer de nouvelles. Si elle est faible (soupçon d'insuffisance MMS), l'éventail des modes opératoires possibles est très restreint, ce qui 
peut conduire à une hyper-sollicitation de certaines fonctions, et/ou à la présence d'activités empêchées entrainant des détériorations du rapport au travail et du collectif, des difficultés d'atteinte de performance et des atteintes possibles à la santé. Enfin, à l'extrême, si elle est absente (insuffisance MMS) on observe un échec quotidien dans l'atteinte des objectifs, même au prix d'une mobilisation coûteuse de la santé (Coutarel et al., 2015 ; Coutarel \& Petit, 2013 ; St-Vincent et al., 2014).

Plusieurs modèles existent dans la littérature pour présenter le principe de régulation et de marge de manœuvre (Aptel \& Vézina, 2008 ; Franchi, 1997 ; Guérin, et al., 1997 ; Leplat \& Cuny, 1977; Sauter \& Swanson, 1996). Vézina en 2001 présente le modèle de l'activité centrée sur la personne en activité (St-Vincent et al., 2014 ; Vézina, 2001), où l'on retrouve les déterminants de l'activité (internes et externes) et les 3 composantes représentatives de la MMS : les stratégies de réalisation développées par l'individu pour faire face aux contraintes, les effets de l'activité sur la santé et les effets sur la performance (Coutarel \& Petit, 2013 ; Coutarel, Roquelaure, \& Daniellou, 2013 ; StVincent et al., 2014 ; Vézina, 2001). Le lien entre ce modèle théorique issu de la pratique de l'ergonomie et la prévention des TMS a été démontré à plusieurs reprises (Caroly, Coutarel, Escriva, Roquelaure, Schweitzer, \& Daniellou, 2008 ; Ouellet \& Vézina, 2014 ; Vézina, 2001). Ce modèle est aujourd'hui décliné dans plusieurs domaines de la prévention des TMS comme la réadaptation (Durand et al., 2008), la conception des systèmes (Lux, El-Mouaini, Daille-Lefevre, Savin, Etienne, \& Siadat, 2017) ou encore l'encadrement de proximité (Cuny-Guerrier, 2017 ; Cuny-Guerrier, Caroly, Coutarel, \& Aublet-Cuvelier, 2015). L'intérêt essentiel de l'approche des TMS par la MMS est d'intégrer des informations sur les ressources dont dispose l'opérateur à l'évaluation plus classique des contraintes auxquelles il est exposé (Coutarel et al., 2015 ; Guérin et al. , 1997 ; Koukoulaki, 2014), pour appréhender l'insuffisance (absence de mode opératoire satisfaisant), le soupçon d'insuffisance (un seul mode opératoire) la suffisance (au moins deux modes opératoires satisfaisants), et l'ampleur de cette suffisance (nombre de modes opératoires satisfaisants disponibles).

7 Dans une précédente étude, nous avons utilisé ce modèle pour connaître la prise en compte actuelle de la régulation de l'activité et MMS par les outils simples d'évaluation du risque TMS (Norval, Zare, Brunet, Coutarel, \& Roquelaure, 2017a). Pour cela, 49 indicateurs représentatifs du modèle de Vézina (2001) ont été caractérisés, tels que présentés dans la figure 1. Cette étude a montré qu'aucun des outils simples de notre échantillon, actuellement utilisé pour l'évaluation du risque TMS en entreprise, ne permet de statuer sur une suffisance, un soupçon d'insuffisance ou une insuffisance MMS et encore moins sur une ampleur de MMS, c'est-à-dire le nombre de stratégies opératoires dont l'opérateur dispose pour faire face à une situation de manière satisfaisante (Norval et al., 2017a ; Norval, Zare, Brunet, Coutarel, \& Roquelaure, 2017b).

Le travail présenté ici vise à étudier comment la mesure de l'état de suffisance de MMS, lors de la phase initiale d'évaluation du risque de TMS, peut utilement enrichir le ciblage des situations critiques et ainsi répondre aux difficultés évoquées (représentativité, validité et fiabilité des outils simples). Il s'agit d'une étude exploratoire en situation réelle de travail qui doit permettre de caractériser la MMS à partir d'informations semi-quantitatives (obtenues par observation) et qualitatives (obtenues par entretien), puis de comparer ces deux approches. 
Figure 1 : Modèle de l'activité centré sur la personne de Vézina (2001) et distinction des termes : composantes, indicateurs de la situation de travail, indicateurs des déterminants, et indicateurs de la Marge de Manœuvre Situationnelle.

Figure 1: Human-centered model of work situations focused on the individuals and their work activity (Vézina, 2001) and distinguishing between components, work situation indicators, determinant indicators, and indicators of situational operational leeway

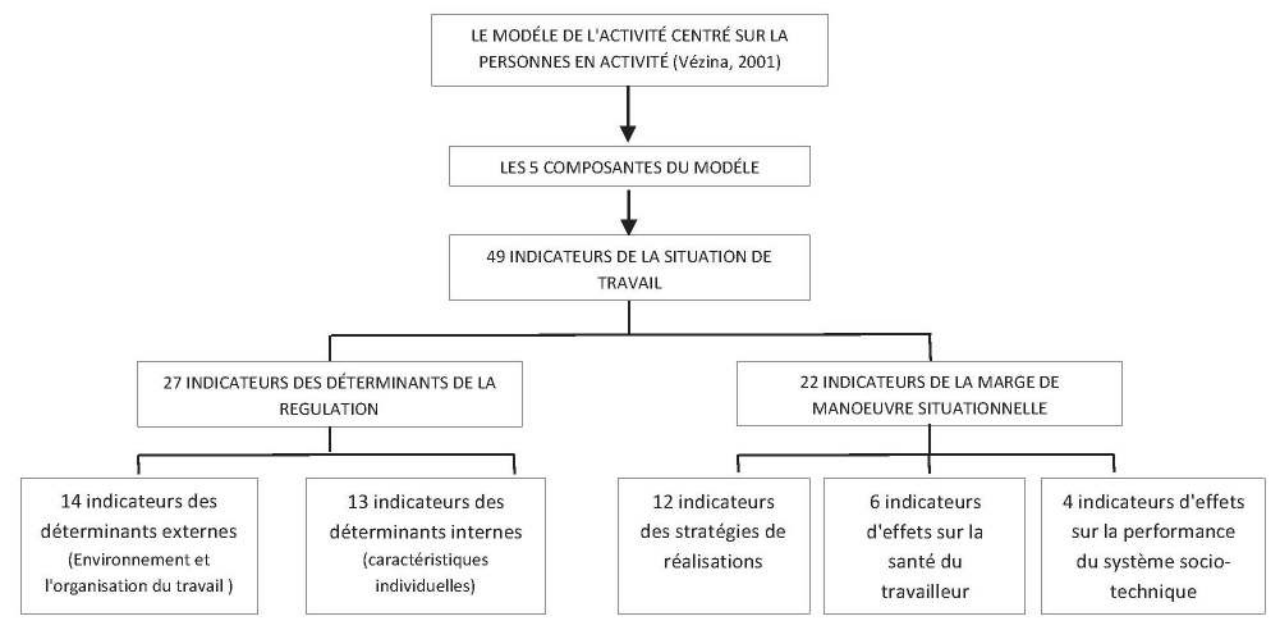

\section{Méthodologie}

\subsection{Contexte d'étude}

Le site de préparation de moteurs d'engins agricoles non routiers (Loiret, Dép. quarante-cinq), terrain de cette étude, comprend plusieurs secteurs de production organisés autour des chaines d'usinages des pièces maitresses du moteur (culasses, blocs moteurs, bielles) et d'une chaine d'assemblages et d'habillages des moteurs complets. Le site permet l'assemblage d'une très forte diversité de produits allant jusqu'à environ 350 modèles de moteurs différents. Cette caractéristique, que l'on retrouve dans plusieurs industries, est un avantage dans l'approche clients (vers le surmesure), mais se traduit par une variabilité constante sur les situations de travail qui apparait comme une difficulté pour les préventeurs lors de la recherche des situations critiques en prévention des TMS. Sur un échantillon de 10 postes (le poste et sa situation de référence), nous avons exploré les indicateurs représentant la MMS lors d'un entretien au poste, pour déterminer l'état de suffisance de MMS (Norval et al., 2017a). L'échantillon de postes a été sélectionné, en accord avec les préventeurs de l'entreprise, comme étant représentatif des difficultés rencontrées par les travailleurs sur les différents secteurs d'activité. Le chercheur qui a recueilli les données sur le terrain a une connaissance approfondie des cas d'études sélectionnés, car il est ergonome-intervenant chargé des questions de prévention des TMS depuis trois ans dans l'entreprise. En amont de l'expérimentation, l'ergonome-intervenant et les opérateurs définissent la situation de référence de chaque poste. La situation de référence représente la situation la plus à risque, en termes de TMS, parmi l'ensemble des situations rencontrées sur le poste de travail. La situation la plus à risque est identifiée suite à l'observation de l'ensemble des situations qui composent le poste avec l'outil simple interne à l'entreprise et suite à un entretien au poste avec l'opérateur. Le chercheur sollicite les représentations de l'opérateur pour connaître quelle est la 
situation qui lui pose le plus de difficultés en termes d'engagement physique, de contraintes organisationnelles, de rapidité d'exécution et celle où il rencontre des aléas fréquents. La situation remontée par l'opérateur est associée aux observations libres réalisées par le chercheur pour confirmer le choix de la situation de référence en amont de l'expérimentation. Pour chaque cas de notre échantillon, nous faisons une distinction entre les postes et les situations de travail de référence sélectionnées sur ce même poste pour explorer la MMS. Les 10 cas étudiés correspondent ainsi aux 10 situations de référence retenues sur les 10 postes (Tableau 1).

10 L'échantillon comprend quatre postes à l'assemblage final du moteur (" assemblage faisceaux moteur", "préparation kits injecteurs", "préparation sous ensemble collecteur/turbo » et "final ligne d'assemblage moteur »), deux postes à l'usinage des bielles (« ébauche » et « finition bielles »), deux postes dans la zone de préparation des kits moteurs (" emballage visseries » et "préparation commande de petites pièces »), un poste dans la zone peinture moteur («marouflage avant peinture»), et un poste dans la zone de préparation moteur avant essai ("équipement/dés-équipement moteur »). Sur les 10 postes sélectionnés, les 10 travailleurs retenus sont des travailleurs considérés par cette entreprise comme expérimentés (étant dans l'entreprise depuis plus de six mois), sans historique récent de maladie professionnelle (cependant 2 opérateurs ont exprimé, pendant l'entretien, qu'ils ont eu une maladie professionnelle sur un autre poste il y a plus de $10 \mathrm{ans}$ ), et volontaires pour participer à cette étude (Tableau 1).

Tableau 1 : Description des cas d'études et des caractéristiques individuelles des opérateurs. Table 1: Description of individual characteristics and case studies

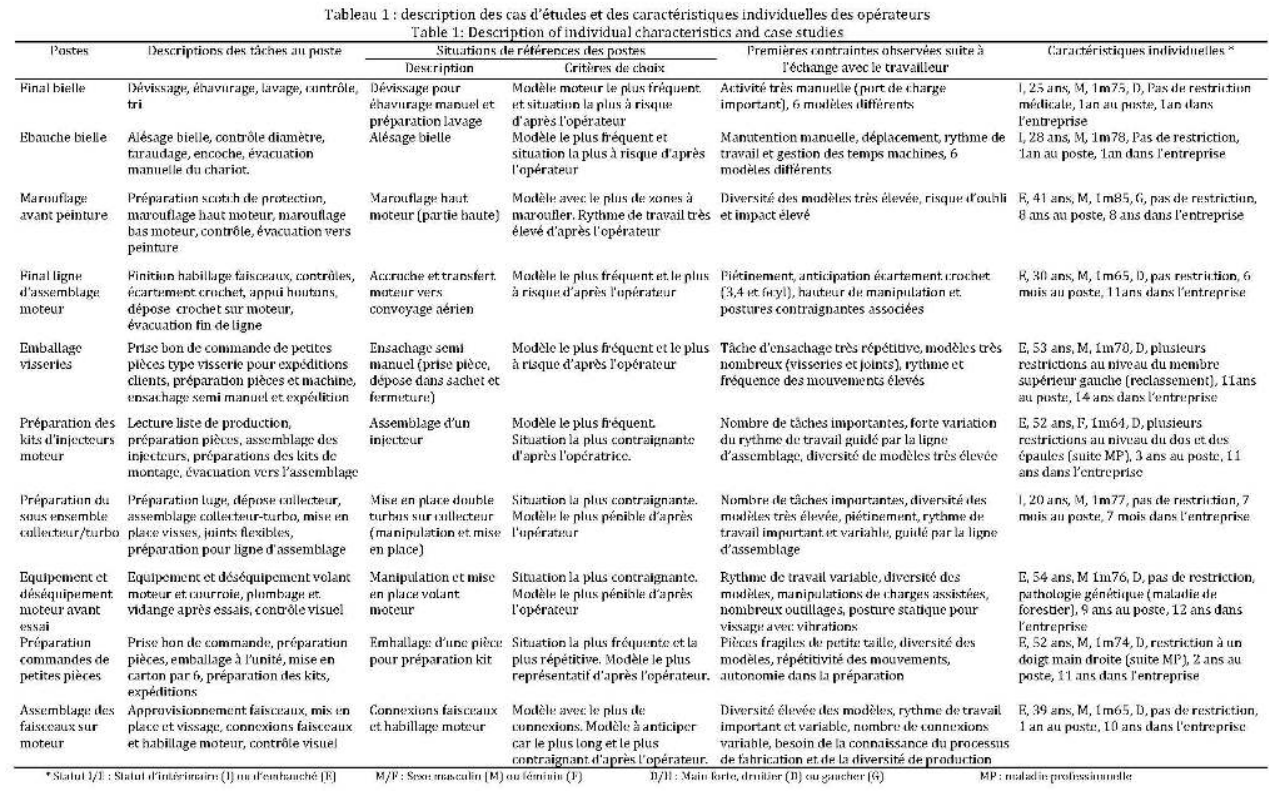

11 Lors de l'expérimentation, l'observation du poste, pendant $1 \mathrm{~h}$ à $1 \mathrm{~h} 30$ sur au moins 10 temps de cycle (durée du cycle de 2,30 min maximum), et l'échange avec le travailleur ont permis la sélection des situations de références. Puis, sur cette situation de référence de chaque poste, les indicateurs MMS ont été explorés pendant $1 \mathrm{~h} 30$ à $2 \mathrm{~h}$ à l'aide d'une grille d'entretien avec l'opérateur déployé en activité. Le temps d'analyse et d'interprétation des données semi-quantitatives étaient approximativement de 
$30 \mathrm{~min}$ (plus ou moins $10 \mathrm{~min}$ ) par situation (calcul des scores de chaque composante et interprétation). L'étude et l'interprétation de la MMS avec les données qualitatives étaient d'environ à $1 \mathrm{~h}$ par situation (plus ou moins $15 \mathrm{~min}$ ). Ce temps représente la retranscription des données et l'interprétation en collectif des signes et indices positifs ou négatifs.

Les conditions d'étude comme la méthode de sélection de la situation de référence parmi l'ensemble des situations particulières du poste, l'évaluateur, la grille d'entretien sur les indicateurs MMS et la méthodologie d'approche étaient volontairement identiques pour chacun des 10 cas d'études.

\subsection{Développement de la grille d'entretien sur les indicateurs de la Marge de Manœuvre Situationnelle}

13 Les indicateurs représentatifs du modèle de Vézina (2001), développés dans une précédente étude, ont été réutilisés pour explorer l'état de suffisance MMS de notre échantillon de situation de travail (Norval et al., 2017a). À partir de questions existantes dans la littérature scientifique et avec l'équipe de recherche en ergonomie, nous avons retravaillé et validé la formulation de chacune des questions pour créer une grille d'entretien libre spécifique MMS. Celui-ci est utilisé comme support d'aide à l'exploration des indicateurs des 3 composantes de la MMS présentés tableau 2. Les indicateurs des déterminants internes (comme par exemple l'expérience et le savoirfaire, les caractéristiques anthropométriques ou l'âge et les capacités physiques) et les indicateurs des déterminants externes (comme par exemple l'organisation globale du travail, les attentes de l'employeur, les moyens mis à disposition et les possibilités organisationnelles et matérielles) sont écartés de l'exploration, et ne sont donc pas intégrés à la grille d'entretien MMS. Nous considérons en effet que l'identification des déterminants à l'origine de la suffisance, du soupçon d'insuffisance ou de l'insuffisance de MMS relève du diagnostic ergonomique, autrement dit de l'analyse ergonomique du travail qui doit suivre l'identification des situations à risque justifiant des investigations approfondies. Les indicateurs représentants les déterminants (internes et externes) pourront être étudiés uniquement lors de l'investigation approfondie pour identifier les pistes d'actions. Il n'est pas du ressort de l'étape d'évaluation du risque de TMS de caractériser ces déterminants. Il nous apparait enfin illusoire de croire qu'un outil simple pourrait réaliser le diagnostic ergonomique de la situation de travail. 
Tableau 2 : Indicateurs de la Marge de Manœuvre Situationnelle, extraits des 49 indicateurs de modèle centré sur la personne en activité (Norval et al., 2017a, 2017b).

Table 2: Indicators of situational operational leeway, extracted from 49 indicators of the human-centered in-activity model (Norval et al., 2017)

\begin{tabular}{|c|c|c|}
\hline $\begin{array}{c}\text { Les composantes } \\
\text { de la situation de } \\
\text { travail }\end{array}$ & $\begin{array}{c}\text { Nature des } \\
\text { informations }\end{array}$ & Indicateurs MMS \\
\hline \multirow{2}{*}{$\begin{array}{l}\text { Activité de } \\
\text { travail et } \\
\text { régulation } \\
\text { (travail réel et } \\
\text { comportement } \\
\text { du travailleur) }\end{array}$} & $\begin{array}{l}\text { Contraintes } \\
\text { de l'activité }\end{array}$ & $\begin{array}{l}\text { Indicateurs du processus de régulation: } \\
\quad \text { Niveau de pénibilité des tâches (ex : importance de l'effort cardio-vasculaire) } \\
\text { Nombre et importance des facteurs de risques observés sur la situation de travail (tonnage, } \\
\text { mouvement rapide et répétitifi, postures contraignantes] }\end{array}$ \\
\hline & $\begin{array}{l}\text { Stratégies de } \\
\text { réalisation }\end{array}$ & 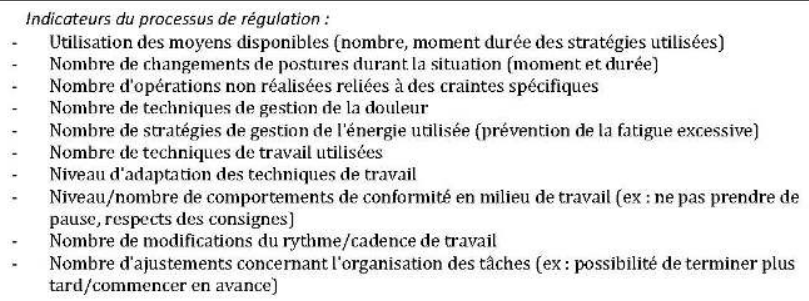 \\
\hline $\begin{array}{l}\text { Effet de la } \\
\text { situation de } \\
\text { travail }\end{array}$ & Santé & $\begin{array}{l}\text { Indicateur des effets de l'activité : } \\
\quad \text { Niveau de douleur en lien avec la situation de travail observée } \\
\text { Niveau/quantité de sommeil * } \\
\text { Nombre de symptômes autres que la douleur en lien avec la situation de travail (ex : sensation } \\
\text { de lourdeur, engourdissement, crampes) } \\
\text { Présence d'une nouvelle lésion apparue pendant ou après la réalisation de la situation de } \\
\text { travail observée } \\
\text { Qunantié de médicaments } \\
\text { Niveau de stress perçu par l'individu et engendré par la situation de travail }\end{array}$ \\
\hline $\begin{array}{l}\text { Effet de la } \\
\text { situation de } \\
\text { travail }\end{array}$ & Performance & $\begin{array}{l}\text { Indicateur des effets de l'activité : } \\
\quad \text { Durée : respect des temps de cycles sur la situation de travail } \\
\quad \text { Respect des délais de production mensuelte ou annuelle } \\
\quad \text { Quantité des produits réalisés par rapport à l'objectif de production alloué à la situation de } \\
\text { travail } \\
\quad \text { Qualité des produits réalisés sur la situation de travail }\end{array}$ \\
\hline
\end{tabular}

\subsection{Méthode d'exploration des indicateurs de marge de manœuvre situationnelle}

\section{Exploration par méthode semi-quantitative}

L'évaluateur a observé les indicateurs des trois composantes de la MMS (Tableau 2) sur chaque situation de référence pour statuer sur la suffisance, la possible insuffisance ou l'insuffisance MMS. Cette observation, à l'aide d'une grille sur les indicateurs de la MMS, a permis d'obtenir un score à quatre niveaux $(0,1,2,3:$ le score le plus bas étant le plus pénalisant en termes de MMS). Ces informations ont été recherchées sur 12 indicateurs pour la composante du processus de réalisation, sur quatre indicateurs pour la composante d'effets directs sur la santé et sur trois indicateurs pour la composante d'effets sur la performance (en gris dans le Tableau 2). Certains indicateurs des composantes d'effets (qualité du sommeil, quantité de médicaments et respect des délais de production mensuelle/annuelle) ont été écartés, car sensibles à des dimensions qui ne révèlent pas forcément de la MMS (en noir dans le Tableau 2).

La retranscription des scores obtenus (données semi-quantitatives) pour les indicateurs MMS a été réalisée pour explorer l'état de suffisance MMS pour chacune des 10 situations de travail de référence. La somme des scores a été calculée séparément pour les trois composantes. Pour chaque composante étudiée, nous avons créé une échelle d'interprétation relative à trois niveaux (score faible, intermédiaire et élevé) en fonction de la proximité des scores de nos 10 situations de référence. Le classement a été réalisé de manière arithmétique en observant la répartition des scores des 
10 situations de référence. La distribution effective constatée autour de trois fourchettes de scores assez distinctes a ainsi permis de relever que les scores « intermédiaires » sont les plus fréquents et que les scores «faibles » et « élevés » sont les moins fréquents. Pour la composante du processus de régulation 12 indicateurs sont interrogés pour un score allant de 0 à 36 pts avec une distribution en trois niveaux : score faible $<20$ pts, score intermédiaire entre $\geq 20$ et $\leq 22$ pts et score élevé $>22$ pts. Pour la composante des effets directs sur la performance, 3 indicateurs sont interrogés pour un score de 0 à $9 \mathrm{pts}$, les scores $\leq 4$ étant considérés comme faibles, les scores entre $>4$ et $\leq 6$ pts comme intermédiaires et les scores $>6$ pts comme élevés. Enfin, pour la composante des effets directs sur la santé, 4 indicateurs sont interrogés pour un score de 0 à 12 pts avec des scores faibles $\leq 6$, des scores intermédiaires entre $>6$ et $\leq 11$ pts et des scores élevés > 11 pts.

L'interprétation générale et le classement des MMS à partir des données semiquantitatives pour chaque situation de travail de référence sont issus des résultats des trois composantes observées séparément :

- Si au moins une des composantes présente un résultat «faible » : insuffisance de MMS pour la situation en question ;

- Si au moins deux composantes présentent des résultats «intermédiaires »: possible insuffisance de MMS ;

- Enfin si au moins deux composantes présentent des résultats « élevés » : suffisance de MMS.

\section{Exploration par méthode qualitative}

17 La reconstitution du modèle de l'activité centré sur la personne est réalisée avec une démarche qualitative pour approfondir les classements des MMS issus des données semi-quantitatives. Sur la base des indicateurs déjà observés dans le cadre de l'analyse semi-quantitative, nous avons réalisé un entretien libre avec chaque opérateur pour obtenir des données qualitatives pour chaque indicateur MMS. Cet entretien permet la reconstitution des composantes de la MMS (processus de régulation, effets directs sur la santé et sur la performance). Nous avons interprété chaque composante en interaction avec les préventeurs de l'entreprise. L'interprétation positive ou négative de chaque composante a été réalisée sur les signes (indices) repérés dans les données qualitatives. À partir des trois composantes observées, le statut de suffisance, de possible insuffisance ou d'insuffisance MMS a été rendu pour chaque situation de travail :

- Si les trois composantes sont négatives, la situation est classée : insuffisance MMS ;

- Si au moins une composante est négative, la situation est classée: possible insuffisance MMS ;

- Enfin, si les trois composantes sont positives, la situation est classée : suffisance MMS.

\subsection{Méthode d'analyse des données}

Les classements des MMS ont été rendus à partir de l'exploration des données semiquantitatives (obtenues par observation) et approfondis par les données qualitatives (obtenues par entretien). Les résultats des deux méthodes d'explorations de la MMS sur les situations de travail seront comparés pour connaître les écarts de jugements et vérifier l'adéquation entre ces deux méthodes d'exploration de l'état de suffisance MMS. 


\section{Résultats}

\subsection{La MMS selon les données semi-quantitatives}

19 Le bilan des trois composantes réalisé pour chacune des situations de référence identifie $50 \%$ des situations de notre échantillon avec une insuffisance MMS (dévissage bielle, accroche moteur sur convoyeur aérien, l'ensacheuse, assemblage d'un injecteur et l'équipement volant moteur), $40 \%$ avec une possible insuffisance (alésage bielle, marouflage haut moteur, sous assemblage collecteur turbo et habillage moteur) et $10 \%$ avec une suffisance de MMS (emballage petites pièces).

Pour exemple on observe au niveau de la situation de dévissage au poste " final bielle », une insuffisance MMS, à partir des scores élevés observés pour les composantes du processus de régulation, des effets directs sur la performance, et du score intermédiaire observé pour les effets directs sur la santé (Tableau 3).

Une interprétation par composante peut être réalisée pour l'ensemble des 10 situations de référence de notre échantillon. Au niveau de la composante des stratégies de réalisation on observe, des scores faibles pour $10 \%$ des situations de référence, des scores intermédiaires pour $70 \%$ des situations de référence et des scores élevés pour $20 \%$ des situations de référence. Au niveau de la composante d'effets directs sur la performance on retrouve $10 \%$ de scores faibles, $70 \%$ de scores intermédiaires et $20 \%$ de scores élevés. Enfin, au niveau de la composante des effets directs sur la performance on identifie $40 \%$ de scores faibles, $50 \%$ de scores intermédiaires et $10 \%$ de scores élevés. Pour les 10 situations de référence on relève la composante effets directs sur la santé comme étant la plus pénalisée par le manque de MMS avec des conséquences négatives pour $40 \%$ des situations de références étudiées.

Tableau 3 : Scores des trois composantes de la Marge de Manœuvre Situationnelle (MMS) à partir des données semi-quantitatives sur les 10 situations de travail étudiées.

Table 3: Semi-qualitative assessment using Situational Operational Leeway (SOL) indicators for the reference situation of ten workstations

\begin{tabular}{|c|c|c|c|c|c|}
\hline \multirow[b]{2}{*}{ Postes de travail } & \multirow[b]{2}{*}{ Situation de référence } & \multicolumn{3}{|c|}{ Somme des indicateurs MMS } & \multirow{2}{*}{$\begin{array}{l}\text { Bilan des résultats: } \\
\text { Suffisance. possible insuffficance, } \\
\text { insuffisance MMS **+* }\end{array}$} \\
\hline & & $\begin{array}{l}\text { Stratégies de } \\
\text { réalisation * }\end{array}$ & $\begin{array}{l}\text { Effets directs sur la } \\
\text { performance }\end{array}$ & $\begin{array}{l}\text { Effets directs sur la } \\
\text { santé }\end{array}$ & \\
\hline Poste final bielle & Dévissage bielle & 17 & 3 & 8 & insuffisance (stratégies et performance) \\
\hline Poste ébauche bielle & Alésage bielle & 26 & 6 & 11 & Possible insuffisance \\
\hline $\begin{array}{l}\text { Poste de marouflage avant } \\
\text { peinture }\end{array}$ & Marouflage haut moteur & 22 & 5 & 11 & Possible insuffisance \\
\hline $\begin{array}{l}\text { Poste final ligne } \\
\text { d'assemblage moteur }\end{array}$ & $\begin{array}{l}\text { Accroche moteur sur } \\
\text { convoyeur aérien }\end{array}$ & 20 & 6 & 6 & Insuffisance (santé) \\
\hline Poste emballage visseries & Ensachage visseries & 21 & 9 & 5 & Insuffisance (santé) \\
\hline $\begin{array}{l}\text { Poste préparation kits } \\
\text { injecteurs moteur }\end{array}$ & Assemblage d'un injecteur & 16 & 6 & 4 & Insuffisance (activité et santé) \\
\hline $\begin{array}{l}\text { Poste préparation sous } \\
\text { ensemble collecteur/turbo }\end{array}$ & $\begin{array}{l}\text { Mise en place du turbo sur } \\
\text { collecteur }\end{array}$ & 21 & 5 & 11 & Possible insuffisance \\
\hline $\begin{array}{l}\text { Poste des equipements/ } \\
\text { équipements avant essai }\end{array}$ & $\begin{array}{l}\text { Equupement volant moteur } \\
\text { avant essai }\end{array}$ & 22 & 6 & 2 & insuffisance (santé) \\
\hline $\begin{array}{l}\text { Poste préparation } \\
\text { commande petites pièces }\end{array}$ & $\begin{array}{l}\text { Emhallage d'une petite } \\
\text { piece }\end{array}$ & 24 & 7 & 10 & suffisance \\
\hline $\begin{array}{l}\text { Poste d'assemblage } \\
\text { faisceaux sur moteur }\end{array}$ & $\begin{array}{l}\text { Habillage et connexion haut } \\
\text { moteur }\end{array}$ & 21 & 5 & 12 & Possible insuffisance \\
\hline
\end{tabular}

$:: 1$. Scores faibles $<20 ; 2$. Scores intermédiaires entre $\geq 20$ et $\leq 22 ; 3.3$ Scores élevés $>22$ (sconre max : 36 points).

**: 1 . Scores faibles $\leq 4: 2$. Scores intermédiaires entre $>4$ et $\leq 6 ; 3$. Scores élevés $>6$ (score max : 9 points).


une possible insuffisance MMS. Si au moins deux colonmes staruent sur un score élevé alors on statue sur une suffisance MMS. 


\subsection{La Marge de Manœuvre Situationnelle selon les données qualitatives}

Pour approfondir les statuts issus des données semi-quantitatives, nous avons reconstitué les trois composantes MMS pour chaque situation de notre échantillon. Pour la situation de dévissage au poste "final bielle ", donnée en exemple figure 2, l'insuffisance MMS prononcée est justifiée à partir des signes (indices) négatifs identifiés au niveau des trois composantes étudiées. Les indices négatifs observés pour la composante des stratégies de réalisation traduisent cette insuffisance de MMS. On retient la faible variabilité des stratégies de réalisation comme représentative de l'impossibilité de faire autrement dans la réalisation de l'activité. Puis nous avons observé la forte variabilité du rythme de travail, marquée par de fortes accélérations ou ralentissements, en fonction de la diversité de produits à réaliser. Cette insuffisance MMS est aussi traduite par la faible prise en compte des propositions d'amélioration faites par l'individu pour faciliter le développement de ses stratégies de réalisation face à une tâche, ou encore par le faible niveau d'échanges de bonnes pratiques et d'entraide avec ses collègues proches qui ne facilitent pas l'accompagnement du travail lors de situations complexes. Au niveau des effets de l'activité, on identifie des douleurs périodiques au niveau des épaules avec une fatigue importante après la réalisation de la situation de travail observée qui traduit les difficultés de réalisation de l'activité. Enfin, au niveau des effets directs sur la performance, on identifie des difficultés pour suivre le rythme de travail (sensation de "travailler dans le retard»), une productivité de la situation de travail périodiquement non atteinte et plusieurs retours négatifs sur la qualité des produits réalisés. 
Figure 2 : reconstitution des trois composantes de la Marge de Manœuvre Situationnelle pour la situation de référence de dévissage bielle (modèle adapté de Vézina, 2001).

Figure 2: Reconstitution of the three components of Situational Operational Leeway indicators for the reference situation of "Connecting rod finishing" (model adapted from Vézina, 2001).

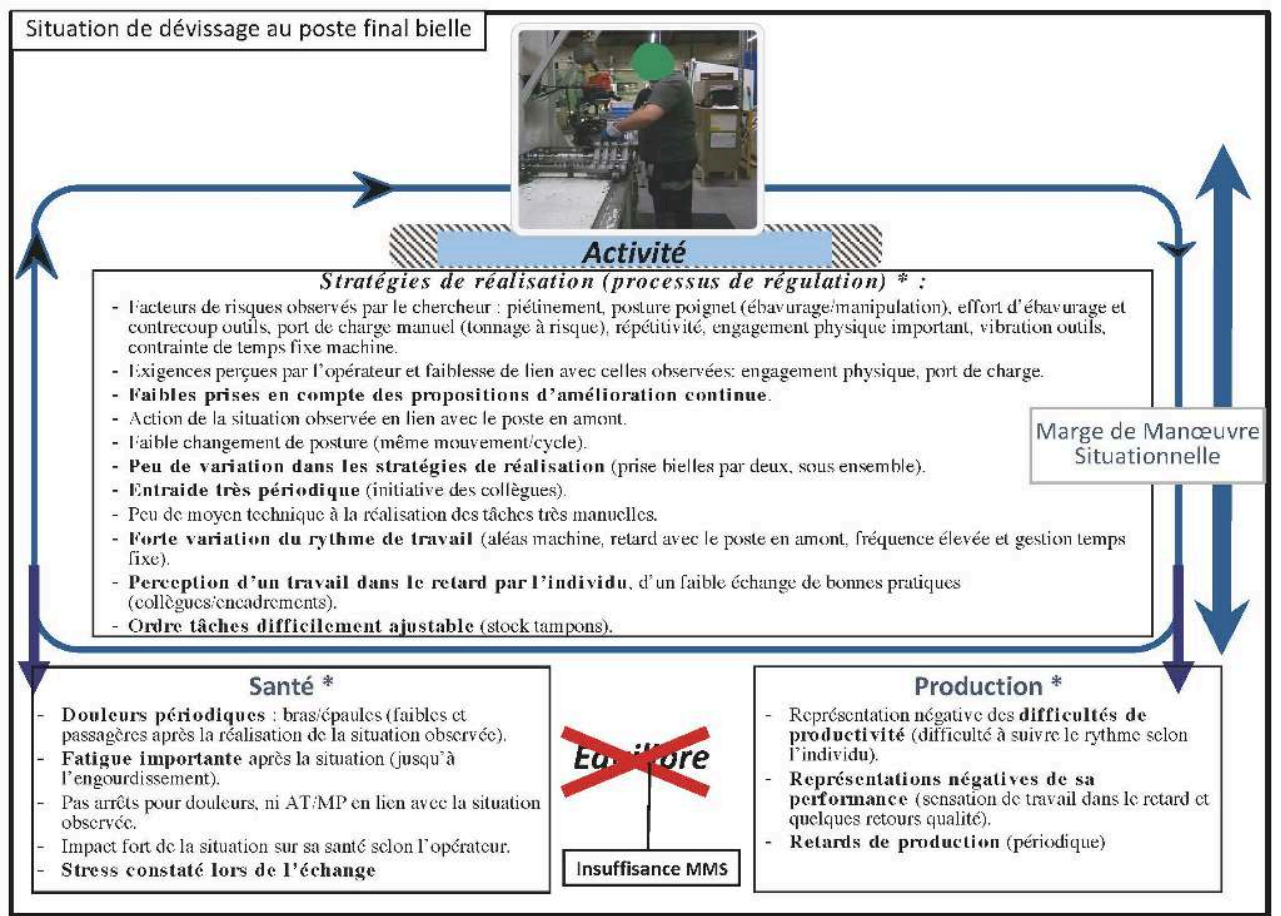

* J.cs indices utilisés pour statuer sont surlignés (en gras) pour les 3 composantes ćtudićes. les situations de travail en suffisance, en possible insuffisance ou en insuffisance MMS. L'interprétation partagée avec les préventeurs de l'entreprise de chacune des trois composantes étudiées a permis de statuer sur une insuffisance MMS pour deux situations de référence (dévissage bielle, et sous assemblage injecteurs), sur une possible insuffisance pour quatre situations de référence (marouflage haut moteur, accroche moteur sur convoyeur aérien, ensachage visserie et la mise en place turbo/ collecteur) et sur une suffisance pour quatre situations de référence (alésage bielle, équipement volant, emballage petites pièces et habillage moteur ; Tableau 4).

Pour chaque situation, la répartition des signes positifs et négatifs en fonction des composantes est différente. En effet, on observe des signes négatifs au niveau des trois composantes pour les situations de dévissage bielle et d'assemblage d'un injecteur. Au contraire, on identifie des signes positifs au niveau des trois composantes pour les situations d'alésage bielle, d'équipement volant moteur, d'emballage de petites pièces et d'habillage/connexions haut moteur. D'autres situations comme le marouflage haut moteur et la mise en place turbo/collecteur présentent des signes positifs pour la composante des stratégies de réalisation et des signes négatifs pour au moins une des deux composantes d'effets (santé ou performance). Enfin, les situations d'accroche moteur sur convoyeur et ensachage visseries présentent des signes négatifs pour les composantes des stratégies de réalisation et d'effets sur la santé alors que les signes sont positifs pour les effets sur la performance.

25 Pour les 10 situations de notre échantillon, on identifie une répartition des signes positifs et négatifs différente d'une composante à l'autre. Au niveau de la composante 
des stratégies de réalisation, on retient 4 statuts négatifs sur 10 situations étudiées avec des indices comme la faible variabilité des stratégies de réalisation, la faible entraide, la forte variabilité du rythme de travail, l'impossibilité de faire autrement ou encore l'impossibilité de changer l'ordre des tâches ou de demander un changement de poste en cas de douleurs. On retient 5 statuts négatifs pour la composante d'effets directs sur la santé avec des informations sur la présence de douleur, de fatigue ou encore la présence de stress durant la réalisation de l'activité. Enfin, pour la composante d'effets sur la performance, 4 statuts sont considérés comme négatifs avec la présence de difficultés liées à l'atteinte des objectifs de performance comme le respect des temps de cycle, la qualité des produits réalisés ou la non-atteinte des objectifs de productivité. Pour les 10 situations de référence on relève la composante effets directs sur la santé comme étant la plus pénalisée par le manque de MMS avec des conséquences négatives pour la moitié des situations de références étudiées.

Tableau 4 : Récapitulatif des résultats de l'exploration qualitative des indicateurs de la Marge de Manœuvre Situationnelle (MMS).

Table 4: The qualitative exploration of Situational Operational Leeway (SOL) indicators



\subsection{Comparaison des statuts issus des données semi-quantitatives et qualitatives}

Nous avons récapitulé les statuts donnés, à partir des scores MMS et de la reconstitution des composantes de la MMS, pour connaître les écarts de jugement entre les deux méthodes d'exploration de l'état de suffisance MMS (écart entre semiquantitative et qualitative). Le tableau 5 montre qu'il y a une différence de statut MMS entre les données qualitatives et semi-quantitatives. Les données qualitatives permettent de confirmer les statuts donnés par les scores pour $50 \%$ des situations de référence de notre échantillon. Pour les $50 \%$ de discordance, les données qualitatives permettent de statuer sur un risque d'apparition des TMS moins élevé qu'avec les données semi-quantitatives. Pour l'ensemble des écarts, les statuts donnés à partir des données semi-quantitatives sont tous déclassés, par les données qualitatives, vers un statut moins à risque. 
On observe des écarts de jugement pour les situations d'ébauche bielle et d'habillage/ connexion moteur avec un statut de possible insuffisance lorsque l'on fait le bilan des scores et un statut de suffisance après avoir recréé les trois composantes de la MMS avec les informations qualitatives. Pour les situations d'accroche moteur sur convoyeur et ensachage visseries, les données semi-quantitatives présentent une insuffisance MMS alors que les données qualitatives aboutissent à une possible insuffisance. Enfin, la situation d'équipement volant moteur avant essais présente une insuffisance MMS d'après les scores et une suffisance MMS d'après les trois composantes MMS.

Tableau 5 : Récapitulatif des statuts de la Marge de Manœuvre Situationnelle (MMS) issu des données semi-quantitatives et qualitatives.

Table 5: Situational Operational Leeway statuses based on semi-quantitative and qualitative data

\begin{tabular}{|c|c|c|c|c|c|c|c|c|c|c|}
\hline Poste & Final bielle & $\begin{array}{l}\text { Ébauche } \\
\text { bielle }\end{array}$ & $\begin{array}{l}\text { Marouflage } \\
\text { avant } \\
\text { peinture }\end{array}$ & $\begin{array}{c}\text { Final ligne } \\
\text { assemblage } \\
\text { moteur }\end{array}$ & $\begin{array}{l}\text { Emballage } \\
\text { visseries }\end{array}$ & $\begin{array}{l}\text { Préparation kits } \\
\text { injecteurs } \\
\text { moteur }\end{array}$ & $\begin{array}{c}\text { Préparation } \\
\text { sous ensemble } \\
\text { collecteur/turbo }\end{array}$ & $\begin{array}{l}\text { Équipements/ } \\
\text { équipements } \\
\text { avant essai }\end{array}$ & $\begin{array}{l}\text { Préparation } \\
\text { commande } \\
\text { petites pièces }\end{array}$ & $\begin{array}{l}\text { Assemblage } \\
\text { faisceaux } \\
\text { moteur }\end{array}$ \\
\hline $\begin{array}{l}\begin{array}{c}\text { Situation de } \\
\text { référence }\end{array} \\
\text { Statuts MMS }\end{array}$ & $\begin{array}{l}\text { Dévissage } \\
\text { bielle }\end{array}$ & $\begin{array}{l}\text { Alésage } \\
\text { bielle }\end{array}$ & $\begin{array}{l}\text { Marouflage } \\
\text { haut moteur }\end{array}$ & $\begin{array}{l}\text { Accroche } \\
\text { moteur sur } \\
\text { convoyeur }\end{array}$ & $\begin{array}{c}\text { Ensachage } \\
\text { visseries }\end{array}$ & $\begin{array}{l}\text { Assemblage d'un } \\
\text { injecteur }\end{array}$ & $\begin{array}{c}\text { Mise en place } \\
\text { turbo sur } \\
\text { collecteur }\end{array}$ & $\begin{array}{l}\text { Equipement } \\
\text { volant moteur } \\
\text { avant essai }\end{array}$ & $\begin{array}{c}\text { Emballage } \\
\text { d'une petite } \\
\text { pièce }\end{array}$ & $\begin{array}{c}\text { Habillage et } \\
\text { connexion } \\
\text { haut moteur }\end{array}$ \\
\hline $\begin{array}{l}\text { Données semi- } \\
\text { quantitatives }\end{array}$ & Insuffisance & $\begin{array}{c}\text { Possible } \\
\text { insuffisance }\end{array}$ & $\begin{array}{c}\text { Possible } \\
\text { insuffisance }\end{array}$ & Insuffisance & Insuffisance & Insuffisance & $\begin{array}{c}\text { Possible } \\
\text { insuffisance }\end{array}$ & Insuffisance & Suffisance & $\begin{array}{c}\text { Possible } \\
\text { insuffisance }\end{array}$ \\
\hline $\begin{array}{c}\text { Données } \\
\text { qualitatives }\end{array}$ & Insuffisance & Suffisance & $\begin{array}{c}\text { Possible } \\
\text { insuffisance }\end{array}$ & $\begin{array}{c}\text { Possible } \\
\text { insuffisance }\end{array}$ & $\begin{array}{c}\text { Possible } \\
\text { insuffisance }\end{array}$ & Insuffisance & $\begin{array}{c}\text { Possible } \\
\text { insuffisance }\end{array}$ & Suffisance & Suffisance & Suffisance \\
\hline $\begin{array}{l}\text { Concordance / } \\
\text { discordance }\end{array}$ & Concordance & Discordance & Concordance & Discordance & Discordance & e Concordance & Concordance & Discordance & Concordance & Discordance \\
\hline
\end{tabular}

\section{Discussion}

\subsection{Dépassement de la vision des contraintes proposée par les « outils simples"}

L'activité de travail mobilise l'individu dans toutes ses dimensions (indissociablement physiques, cognitives, subjectives et sociales) et se construit dans un cadre d'objectifs, de contraintes et de ressources (Coutarel \& Petit, 2013 ; St-Vincent et al., 2014). Or les « outils simples» actuellement utilisés ne permettent pas cette vision globale, car ils s'orientent essentiellement vers l'étude des contraintes/expositions (Malchaire et al., 2011; Zare et al., 2015; Zare, Biau, Croq, \& Roquelaure, 2014). Les stratégies de réalisation étudiées lors de l'exploration de la MMS sont le résultat de la rencontre entre des contraintes (environnementales, organisationnelles et individuelles), et des ressources que l'individu utilise pour faire face à l'activité et à ces variabilités (Coutarel et al., 2015 ; Coutarel \& Petit, 2013 ; Cuny-Guerrier, 2017 ; Vézina, 2001). Au niveau de la composante des stratégies de réalisation, les ressources de l'activité permettant à l'individu la gestion des variabilités (internes et externes) sont prises en compte lors de l'exploration de l'état de suffisance MMS. Nous retrouvons par exemple la prise en compte de la possibilité de varier ses stratégies de réalisation traduites par l'entraide, l'inversion de l'ordre des tâches modifiables, la maîtrise du rythme de travail par l'individu, ou encore l'utilisation des moyens d'aide à disposition. En complément, les effets directs sur la performance et la santé sont des informations de sortie de la régulation. Ces informations représentent les conséquences des stratégies de réalisation déployées par l'individu pour faire face à la variabilité des conditions de réalisation et à sa propre variabilité (Gaudez, Gilles, \& Savin, 2016; St-Vincent et al., 
2014). L'exploration de l'état de suffisance de MMS propose une évolution dans la recherche des situations critiques, car elle tient compte des ressources de l'activité qui peuvent en certains cas venir « compenser » les contraintes présentes sur la situation de travail. Ceci explique que l'exploration de la MMS conduit à identifier moins de situations critiques que les outils simples habituels, centrés eux sur les expositions (Norval, Zare, Brunet, Coutarel, \& Roquelaure, 2018). Nous proposons de compléter le ciblage actuellement réalisé avec des outils simples par l'exploration de l'état de suffisance MMS.

\subsection{Exploration des indicateurs de la Marge de Manœuvre Situationnelle}

L'exploration des composantes de la MMS apparait être une méthode permettant la sélection des situations les plus critiques. Cependant ce travail d'investigation nécessite d'avoir des compétences spécifiques en ergonomie et une connaissance approfondie des situations de travail. Les deux méthodes d'exploration (qualitative et semiquantitative) présentent les situations de dévissage bielle et d'assemblage d'un injecteur comme les plus à risque parmi les 10 situations de référence étudiées. Ces situations sont considérées à risque à partir des signes qui traduisent les difficultés rencontrées par les individus pour faire face à la diversité de production tout en respectant les exigences du travail (dont le rythme de travail, mais aussi la qualité, la productivité, etc.). Ces signes peuvent être les accélérations/ralentissements importants, les changements fréquents du rythme de travail ou encore l'impossibilité de faire autrement dans les stratégies déployées. Les stratégies de réalisation déployées étant considérées comme à risque, il apparait cohérent de retrouver des effets négatifs sur la santé et la performance, comme des douleurs, de la fatigue importante et/ou l'échec dans l'atteinte des objectifs.

La méthode semi-quantitative permet d'identifier la situation d'emballage d'une petite pièce comme la moins à risque, alors que la méthode qualitative, identifie en plus, les situations d'alésage bielle, d'équipement volant moteur avant essai et d'habillage/ connexion haut moteur. Ces situations ressortent comme à risque faible, car, d'après les indices issus des informations qualitatives, les individus arrivent à faire face aux variabilités de l'activité avec une diversité des stratégies de réalisation qui leur permettent de respecter les objectifs de performance tout en préservant leur santé. La diversité des stratégies de réalisation caractérisant la suffisance MMS permet au travailleur de rechercher la meilleure "façon » de réaliser l'activité en fonction des variabilités rencontrées. Ainsi en fonction des situations de travail rencontrées, l'individu doit pouvoir demander de l'aide en cas de difficultés, avoir la possibilité de maîtriser son rythme et/ou d'inverser l'ordre des tâches afin de maintenir un rythme de travail constant tout en laissant la possibilité de développer de nouvelles stratégies de réalisation.

31 Les données qualitatives permettent de confirmer les statuts issus des données semiquantitatives pour la moitié des cas d'étude. Pour les écarts de jugement rencontrés, les statuts donnés à partir des données semi-quantitatives sont tous déclassés par les informations qualitatives vers un statut moins à risque. Ainsi, la caractérisation de l'état de suffisance MMS, issue des scores, permet une première appréciation du risque d'apparition des TMS, même si son jugement est plus critique (à risque plus élevé) que 
les statuts donnés à partir de la reconstitution des trois composantes MMS. Le classement MMS obtenu avec l'aide des scores (données semi-quantitatives) est réalisé sans mise en relation des informations obtenues et sans pouvoir contextualiser l'interprétation. Chaque indicateur MMS est questionné séparément, ce qui ne permet pas la contextualisation et la mise en relation des informations. C'est pourquoi nous avons choisi de préciser les statuts par une analyse qualitative des trois composantes de la MMS.

Les écarts de jugement observés entre les résultats des données semi-quantitatives et qualitatives, pour $50 \%$ des situations de travail de notre échantillon, peuvent être expliqués par la différence de précision des méthodes d'exploration. Plusieurs améliorations de l'approche avec les données semi-quantitatives peuvent être proposées pour limiter ces écarts de jugement. Nous envisageons par exemple d'augmenter l'impact de certains facteurs d'une même composante en proposant une interprétation des scores avec des pondérations. Nous pouvons aussi proposer de tenir compte du lien entre certains indicateurs MMS. Par exemple, l'impossibilité d'inverser l'ordre des tâches rend difficile l'entraide entre collègues ou encore la variabilité du rythme de travail (accélération/ralentissement) qui complexifie sa maîtrise.

\subsection{Vers une vision globale des stratégies de réalisation et de leurs effets directs}

33 L'interprétation partagée a permis une vision d'ensemble des trois composantes qui augmente la fiabilité du statut obtenu. Les informations obtenues pour chaque composante sont récapitulées dans un tableau qui permet notamment d'imaginer un lien direct entre les stratégies de réalisation déployées et leurs effets sur la performance et la santé. L'exemple de la situation de marouflage haut moteur est représentatif de la vision globale proposée, en effet les indices positifs au niveau des stratégies de réalisation sont à mettre en perspective avec les informations négatives sur les effets de l'activité (santé et performance). En effet, les indices de la composante des stratégies de réalisation laissent penser qu'il y a la présence d'une MMS suffisante (plusieurs stratégies de réalisation, possibilité d'entraide et d'inverser l'ordre des tâches), mais lorsque l'on observe en plus, les signes négatifs pour les effets (douleurs, stress et difficultés d'atteinte des objectifs), nous pouvons supposer que l'individu n'utilise pas la possibilité qu'il a de développer ses propres stratégies de réalisation de manière adaptée. La vision globale des trois composantes MMS est essentielle et indispensable pour juger de l'état de suffisance et pour comprendre le lien entre les stratégies de réalisation déployées par les individus et leurs effets (santé et performance).

\subsection{Prise en compte des variabilités et de la diversité de l'activité de travail}

Les évolutions envisagées pour améliorer la recherche des situations critiques semblent diminuer les effets de la variabilité de l'activité sur les résultats de l'évaluation du risque TMS. La variabilité de production, la diversité des actions à réaliser, la diversité des caractéristiques individuelles et les dimensions collectives du travail ne peuvent pas être prises en considération lors de la caractérisation des risques en situation de 
travail avec les outils simples d'évaluation du risque actuellement utilisés par les préventeurs (Bongers, Ijmker, Van den Heuvel, \& Blatter, 2006; Reed \& Flannagan, 2000 ; Roquelaure et al., 2013; Zare, 2015 ; Zare et al., 2014). Les conclusions de Zare (2015) et Roquelaure et ses collègues (2013) montrent que l'évaluation du risque TMS avec des outils simples doit relever le défi de la prise en compte de la diversité et de la variabilité des tâches à réaliser. Les effets de la variabilité de production, et de la variabilité inter et intra individuelle ne peuvent pas être ignorés par les entreprises et les managers. La volonté de standardisation des situations de travail dans les entreprises ne favorise pas la prise en compte des variabilités. Ceci conduit à sousestimer l'intérêt de la construction d'un panel élargi de stratégies opératoires, et donc tend à figer l'activité dans la répétition d'un même mouvement qui n'est donc jamais vraiment adapté à la situation et ses variabilités. On peut ainsi expliquer la survenue des atteintes de la santé et/ou de la performance (Daniellou \& Rabardel, 2005 ; Durand, Vézina, Baril, Loisel, Richard, \& Ngomo, 2009; Major \& Vézina, 2015). Nous pensons qu'il est important de prendre en compte cette diversité de stratégies comme une information sur le risque d'apparition de la pathologie avec la recherche d'indices sur leurs variabilités (alternance de la «façon de faire » en fonction des contraintes), leurs rythmes (accélérations/ralentissements) ou encore leurs effets. La non-prise en compte de la variabilité du travail et de la diversité des situations conduit à des évaluations peu fiables et sujets à variabilité puisque très dépendantes du moment où se réalise l'évaluation (Bao et al., 2009 ; Chiasson et al., 2012 ; Mathiassen, Liv, \& Wahlström, 2012 ; Takala et al., 2010; Zare et al., 2015). L'approche de la MMS invite à considérer l'importance du choix du ou des moments d'évaluation et de leur représentativité dans le cycle de travail (la plus critique, la moins critique, etc.).

De plus malgré l'enrichissement des connaissances sur les facteurs explicatifs de la pathologie, plusieurs revues d'outils simples montrent que la plupart d'entre eux sont orientés vers la prise en compte des contraintes essentiellement biomécaniques (Chiasson et al., 2012 ; David, 2005 ; Denis, Lortie, \& Rossignol, 2000 ; Malchaire et al., 2011 ; Punnett, Fine, Keyserling, Herrin, \& Chaffin, 2000 ; Takala et al., 2010). Pour faire face aux limites d'utilisation des outils simples et pour dépasser la vision de «cause à effet » des facteurs de risque, la mesure de l'état de suffisance MMS est proposée pour compléter l'approche actuelle et faciliter l'activité de recherche des situations critiques pour les préventeurs en entreprise. Or dans une précédente étude, nous avons montré que les outils simples d'évaluation du risque TMS actuellement utilisés par les préventeurs ne peuvent pas déterminer si la MMS est suffisante pour permettre une régulation de l'activité et donc l'atteinte des objectifs de performance sans impact sur la santé de l'individu (Norval et al., 2017a, 2017b).

\subsection{La MMS une opportunité lors de la recherche des situations critiques}

Les connaissances sur la MMS et le lien avec la prévention des TMS sont aujourd'hui reconnus par les scientifiques; mais peu investis par les praticiens (Cuny-Guerrier, 2017 ; Durand et al., 2008 ; Lux et al., 2017 ; Major \& Vézina, 2011). Une des raisons de ce faible investissement par les praticiens peut être le manque de formalisation, le manque d'objectivation des indicateurs MMS ou encore les difficultés de diffusion, c'est pourquoi nous proposons d'autres études en ce sens. Le modèle de Vézina a été choisi 
comme référence, car nous avons identifié son application dans des domaines variés de la prévention des TMS, ce qui encourage son développement à d'autres domaines comme la recherche des situations critiques. En effet, ce modèle créé pour l'intervention en ergonomie a été repris et ajusté à la réadaptation au travail, la conception ou l'encadrement de proximité (Cuny-Guerrier et al., 2015; Durand et al., 2008 ; Durand et al., 2009 ; Lux et al., 2017).

Juger l'état de suffisance MMS pourrait être une méthode pour compléter le score d'exposition issu des outils simples et comprendre «comment» et avec «quelles conséquences " les individus font face aux contraintes. Les indicateurs MMS utilisés (Tableau 4) sont tirés de notre précédente étude, Norval, et ses collègues (2017), et considérés comme représentant les composantes des stratégies de réalisation et de leurs effets (Coutarel, Daniellou, \& Dugué, 2003 ; Cuny-Guerrier, 2017; Durand et al., 2009 ; Gouédard, \& Rabardel, 2012 ; Major \& Vézina, 2011; Tomás \& Fernandez, 2015). Les indicateurs proposés sont assez larges pour ne pas restreindre les champs d'études et faciliter l'exploration. Nous avons écarté les composantes des déterminants internes et externes, car elles n'apparaissent pas appropriées pour repérer les situations les plus exposantes. En effet, l'analyse ergonomique du travail qui identifie les déterminants sur lesquels agir n'est pas de l'objet de l'évaluation du risque même si un certain nombre d'outils simples dits «d'évaluation des risques» explorent quelques déterminants (Norval et al., 2017a). La mesure de la suffisance de la MMS se concentre sur l'objectif de repérage des situations potentiellement critiques. C'est l'analyse ergonomique du travail qui devra ensuite établir pour ces situations le diagnostic permettant d'identifier les configurations articulant des déterminants majeurs, voire les déterminants des déterminants. Aucun outil simple d'évaluation du risque ne peut tenir cet objectif: les déterminants potentiellement pertinents sont multiples, et par ailleurs ils sont liés. Seule l'analyse ergonomique du travail, de par son approche qualitative, globale et systémique des situations peut répondre à ces exigences (StVincent et al., 2014 ; Vézina, 2001).

\subsection{Limites et perspectives}

38 Dans cette étude la MMS a été explorée sur une seule situation de travail sélectionnée, par le chercheur en collaboration avec le travailleur, comme étant la situation de référence la plus à risque ou la plus contraignante parmi l'ensemble des situations que compose le poste. Ne pas prendre en compte et ne pas gérer la diversité des situations au moment de produire l'évaluation implique qu'on ne sait pas exactement ce que représente la mesure (des situations faciles, moyennes, les plus difficiles ?), il est donc plus pertinent d'évaluer la ou les situations les plus à risques. Dans notre étude la sélection des situations critiques réalisée par l'ergonome-intervenant en collaboration avec les opérateurs est discutable. En effet, si le choix de la situation de référence, réalisé au départ de l'étude, s'était porté sur d'autres situations, lors de l'analyse avec l'ensemble des chercheurs, nous n'aurions peut-être pas trouvé les mêmes résultats.

L'application par les préventeurs en entreprises, de cette méthode de sélection des situations critiques par l'état de suffisance MMS, peut-être une des réponses face aux difficultés actuellement rencontrées lors de l'utilisation d'outils simples d'évaluation du risque TMS. Cependant, les notions de régulation de l'activité et de MMS créent des incompréhensions dans les entreprises, car en plus des difficultés 
d'opérationnalisation, elles comportent des informations subjectives et objectives que les entreprises n'ont pas l'habitude de manipuler. Le manque de littérature sur les indicateurs et les déterminants de la MMS augmentent les incompréhensions et les difficultés d'application, symbolisées par l'absence de ces notions dans les outils d'évaluation du risque TMS (David, 2005; Malchaire et al., 2011; Ringelberg \& Koukoulaki, 2002 ; Stock et al., 2005 ; Takala et al., 2010).

Nous proposons pour la première fois une liste d'indicateurs et une méthodologie pour explorer l'état de suffisance MMS. Cependant la formulation des indicateurs MMS pourrait être améliorée dans le cadre d'études complémentaires afin d'enrichir l'objectivation de l'état de suffisance en situation réelle (fiabilité, répétabilité). Les temps de cycles des situations de travail choisies étaient courts ( 2 min $30 \mathrm{~s}$ maximum). L'exploration sur des temps de cycles longs peut être envisageable, mais d'autres études sont nécessaires pour connaître sa faisabilité.

41 Notons que la grille d'entretien créée à partir des indicateurs MMS est utilisée pour la première fois en situation réelle, celle-ci doit faire l'objet d'une validation (répétabilité, fiabilité) pour pouvoir être réutilisée par les préventeurs en entreprises, lors de la recherche des situations critiques. Le travail de développement et de validation de cette grille d'entretien pourrait être mis en place avec la création d'un groupe de travail composé d'une équipe pluridisciplinaire d'ergonomes, de médecins et d'épidémiologistes pour travailler à la formulation des questions en lien avec les indicateurs développés et identifier les questions déjà existantes dans la littérature. Ce groupe de travail peut envisager d'étudier, à moyen terme, le rôle soupçonné des indicateurs d'insuffisance MMS dans l'apparition des TMS.

\section{Conclusion}

La notion de MMS propose un changement de paradigme. Il ne s'agit pas "seulement » de diminuer les contraintes ou les expositions à certains facteurs de risque, comme cela est généralement proposé, mais de tendre vers une prévention par le développement des acteurs à partir du développement de leurs activités professionnelles (Coutarel \& Petit, 2013). Comme réalisée dans cette étude de cas, l'exploration de l'état de suffisance MMS dès la recherche des situations critiques est envisagée pour améliorer la qualité de l'évaluation du risque TMS. Les TMS sont alors appréhendés comme un échec de la régulation de l'activité par l'individu, qui est le reflet d'un déficit de MMS pour l'opérateur sur son poste de travail. Ce déficit individuel étant lui-même lié à un déficit de la marge de manœuvre de nombreux acteurs de l'entreprise comme le manager de proximité ou l'ingénieur méthode qui participent à la conception des situations de travail.

La MMS est un indicateur de la rencontre entre les contraintes et les ressources de l'opérateur en situation de travail, qui pourrait compléter les contraintes classiquement étudiées par les outils d'évaluation du risque TMS actuellement déployés dans les industries afin d'améliorer le ciblage des situations critiques. Les indicateurs des stratégies de réalisation, complétés par les informations sur les effets directs de l'activité (en termes de santé et de performance) permettent d'augmenter la pertinence et la fiabilité du ciblage réalisé. Deux méthodes de caractérisation de l'état de suffisance MMS (semi-quantitative et qualitative) sont testées sur un même échantillon de situation de travail. La reconnaissance scientifique de la MMS, comme centrale en 
prévention des TMS, et son application dans plusieurs domaines de la prévention est une opportunité pour améliorer la recherche des situations critiques en entreprises. Cette étude propose d'enrichir le ciblage des situations critiques par la prise en compte de l'état de suffisance MMS en complément des outils simples d'évaluation du risque TMS actuellement utilisés.

\section{BIBLIOGRAPHIE}

Albert, V., Vézina, N., Bilodeau, H., \& Coutarel, F. (2017). Comment et pourquoi : proposition d'un modèle d'évaluation des processus de la phase de développement d'interventions ergonomiques. Cadre méthodologique d'une évaluation de la phase de développement d'interventions ergonomiques. Perspectives interdisciplinaires sur le travail et la santé, 19(3).

Aptel, M., Lafaurie, S., Tronchet, L., \& Atain-Kouadio, J. J. (2000). OREGE : un outil simple d'évaluation des facteurs de risque biomécaniques de TMS du membre supérieur. Les notes scientifiques et techniques de l'INRS, (NS 196), p. 122.

Aptel, M., \& Vézina, N. (2008). Quels modèles pour comprendre et prévenir les TMS. Pour une approche holistique et dynamique, 2. Actes du $2^{e}$ Congrès francophone sur les troubles musculosquelettiques: de la recherche à l'action, Groupe de recherche francophone sur les troubles musculosquelettiques, IRSST : Montréal.

Armstrong, T. J., Buckle, P., Fine, L. J., Hagberg, M., Jonsson, B., Kilbom, A., \& Viikari-Juntura, E. R. (1993). A conceptual model for work-related neck and upper-limb musculoskeletal disorders. Scandinavian journal of work, environment \& health, 19(2), 73-84.

Badets, P., Merlo, C., \& Pilniere, V. (2017). Prise en compte de l'efficience humaine pour pallier les limites du Lean. Actes du $12^{e}$ congrès international de génie industriel, Compiègne : France.

Bao, S., Howard, N., Spielholz, P., Silverstein, B., \& Polissar, N. (2009). Interrater reliability of posture observations. Human Factors, 51(3), 292-309.

Barrero, L. H., Katz, J. N., \& Dennerlein, J. T. (2009). Validity of self-reported mechanical demands for occupational epidemiologic research of musculoskeletal disorders. Scandinavian journal of work, environment \& health, 35(4), 245-260.

Bodin, J., Garlantézec, R., Costet, N., Descatha, A., Fouquet, N., Caroly, S., \& Roquelaure, Y. (2017). Forms of work organization and associations with shoulder disorders : results from a French working population. Applied Ergonomics, 59, 1-10.

Bongers, P., Ijmker, S., Van den Heuvel, S., \& Blatter, B. (2006). Epidemiology of work related neck and upper limb problems: psychosocial and personal risk factors (part I) and effective interventions from a bio behavioural perspective (part II). Journal of occupational rehabilitation, 16(3), 272-295

Bourgeois, F., \& Hubault, F. (2005). Prévenir les TMS. De la biomécanique à la revalorisation du travail, l'analyse du geste dans toutes ses dimensions. Activités, 2(1). https:// journals.openedition.org/activites/1561 
Bouville, G. (2013). Les effets de la lean production sur les tms et les arrêts maladie : les résultats d'une étude de cas rétrospective dans une entreprise de maintenance ferroviaire. Travailler, 29(1), 183-202.

Caroly, S., Coutarel, F., Escriva, E., Roquelaure, Y., Schweitzer, J., \& Daniellou, F. (2008). La prévention durable des TMS. Rapport d'étude pour la Direction Générale du Travail. Anact, dossier thématique TMS.

Caroly, S., Simonet, P., \& Vézina, N. (2015). Marge de manœuvre et pouvoir d'agir dans la prévention des TMS et des RPS. Le travail humain, 78(1), 1-8.

Chiasson, M.-È., Imbeau, D., Aubry, K., \& Delisle, A. (2012). Comparing the results of eight methods used to evaluate risk factors associated with musculoskeletal disorders. International Journal of Industrial Ergonomics, 42(5), 478-488.

Coutarel, F. (2004). La prévention des troubles musculo-squelettiques en conception : quelles marges de manœuvre pour le déploiement de l'activité ? Thèse en ergonomie non publiée, Université Victor Segalen, Bordeaux.

Coutarel, F., Caroly, S., Vézina, N., \& Daniellou, F. (2015). Marge de manœuvre situationnelle et pouvoir d'agir : des concepts à l'intervention ergonomique. Le Travail Humain, 78(1), 9-29.

Coutarel, F., Daniellou, F., \& Dugué, B. (2003). Interroger l'organisation du travail au regard des marges de manœuvre en conception et en fonctionnement. La rotation est-elle une solution aux TMS ?. Conception et organisation du travail dans les abattoirs en France. Perspectives interdisciplinaires sur le travail et la santé, 5(2).

Coutarel, F., Daniellou, F., \& Dugué, B. (2005). La prévention des troubles musculo-squellettiques : quelques enjeux épistémologiques. Activités, 2(1), 3-19. https://journals.openedition.org/ activites/1550

Coutarel, F., \& Petit, J. (2013). Prévention des TMS et développement du pouvoir d'agir. Dans Falzon, P. (Ed.), Ergonomie constructive (pp. 175-190). Paris : Presses Universitaires de France.

Coutarel, F., Roquelaure, Y., \& Daniellou, F. (2013). Le défi ergonomique face aux TMS d'origine professionnelles. Ergonomie : travail, conception, santé, 255-267.

Cuny-Guerrier, A. (2017). Régulations et marges de manœuvre situationnelles des encadrants de proximité en sous-traitance : un enjeu de prévention des TMS. Thèse en ergonomie, Université Grenoble Alpes, Grenoble.

Cuny-Guerrier, A., Caroly, S., Coutarel, F., \& Aublet-Cuvelier, A. (2015). Quelle prévention des TMS dans l'activité de l'encadrement de proximité en sous-traitance interne ? Un cas dans le secteur de la découpe de viande. Perspectives interdisciplinaires sur le travail et la santé, 17(2), 1-23.

Daniellou, F., \& Rabardel, P. (2005). Activity-oriented approaches to ergonomics : some traditions and communities. Theoretical issues in Ergonomics science, 6(5), 353-357.

Davezies, P. (2007). Intensification. Danger : le travail rétréci. Santé et travail, 57, 30-33.

David, G. (2005). Ergonomic methods for assessing exposure to risk factors for work-related musculoskeletal disorders. Occupational medicine, 55(3), 190-199.

Denis, D., Lortie, M., \& Rossignol, M. (2000). Observation procedures characterizing occupational physical activities : critical review. International journal of occupational safety and ergonomics, $6(4)$, 463-491. 
Durand, M., Vézina, N., Baril, R., Loisel, P., Richard, M., \& Ngomo, S. (2008). La marge de manœuvre de travailleurs pendant et après un programme de retour progressif au travail : définition et relations avec le retour à l'emploi. Montréal : RSST, R-566.

Durand, M.-J., Vézina, N., Baril, R., Loisel, P., Richard, M.-C., \& Ngomo, S. (2009). Margin of manoeuvre indicators in the workplace during the rehabilitation process : a qualitative analysis. Journal of occupational rehabilitation, 19(2), 194-202.

Falzon, P. (Ed.). (2013). Ergonomie constructive. Paris : Presses Universitaires de France.

Franchi, P. (1997). Agir sur les maladies professionnelles : l'exemple des troubles musculosquelettiques (TMS). Réseau Anact, Ed. Liaisons, p. 61.

Gaudez, C., Gilles, M., \& Savin, J. (2016). Intrinsic movement variability at work. How long is the path from motor control to design engineering? Applied Ergonomics, 53, 71-78.

Gollac, M., \& Volkoff, S. (1996). Citius, altius, fortius [L'intensification du travail]. Actes de la recherche en sciences sociales, 114(1), 54-67.

Gouédard, C., \& Rabardel, P. (2012). Pouvoir d'agir et capacités d'agir : une perspective méthodologique ? Illustration dans le champ de la santé, sécurité et conditions de travail. Perspectives interdisciplinaires sur le travail et la santé, 14(2).

Guérin, F., Laville, A., Daniellou, F., Duraffourg, J., \& Kerguelen, A. (1997). Comprendre le travail pour le transformer : la pratique de l'ergonomie. Lyon-Montrouge : Ed. de l'Anact.

Hagberg, M., Kuorinka, I., \& Forcier, L. (1995). Les lésions attribuables au travail répétitif. (LATR) : Éditions MultiMondes.

Karsh, B.-T. (2006). Theories of work-related musculoskeletal disorders : Implications for ergonomic interventions. Theoretical issues in Ergonomics science, 7(1), 71-88.

Koukoulaki, T. (2014). The impact of lean production on musculoskeletal and psychosocial risks: An examination of sociotechnical trends over 20 years. Applied Ergonomics, 45(2), 198-212.

Leplat, J. (2006). La notion de régulation dans l'analyse de l'activité. Perspectives interdisciplinaires sur le travail et la santé, 8(1).

Leplat, J., \& Cuny, X. (1977). Introduction à la psychologie du travail. Paris : Presses universitaires de France.

Lux, A., El-Mouaini, I., Daille-Lefevre, B., Savin, J., Etienne, A., \& Siadat, A. (2017). Santé et sécurité au travail : quatre approches en conception pour spécifier et simuler des marges de manœuvre pour les futurs opérateurs de production. Actes du congrès International de génie industriel (GIGI), Compiègne, France.

Major, M-E., \& Vézina, N. (2011). Élaboration d'un cadre de référence pour l'étude des stratégies : analyse de l'activité et étude de cas multiples dans deux usines de crabe. Perspectives interdisciplinaires sur le travail et la santé, 13(2).

Major, M.-E., \& Vézina, N. (2015). Analysis of worker strategies: A comprehensive understanding for the prevention of work related musculoskeletal disorders. International Journal of Industrial Ergonomics, 48, 149-157.

Malchaire, J., Gauthy, R., Piette, A., \& Strambi, F. (2011). Classification de méthodes d'évaluation et/ou de prévention des risques de troubles musculosquelettiques. Bruxelles : European Trade Union Institute. 
Mathiassen, S. E. (2006). Diversity and variation in biomechanical exposure: what is it, and why would we like to know? Applied Ergonomics, 37(4), 419-427.

Mathiassen, S. E., Liv, P., \& Wahlström, J. (2012). Cost-efficient observation of working postures from video recordings-more videos, more observers or more views per observer? Work, 41, 2302-2306.

Norval, M., Zare, M., Brunet, R., Coutarel, F., \& Roquelaure, R. (2018). Outils simples d'évaluation $\mathrm{du}$ risque TMS et exploration des indicateurs de la marge de manœuvre en situation réelle du travail. Actes du 53e congrès de la SELF Bordeaux, pp. 836-848.

Norval, M., Zare, M., Brunet, R., Coutarel, F., \& Roquelaure, Y. (2017a). Operational leeway in the work situations: Do ergonomic risk assessment tools consider operational leeway for job analysis ? International Journal of Occupational Safety and Ergonomics, 25(3), 1-29. https://doi.org/ $10.1080 / 10803548.2017 .1387392$

Norval, M., Zare, M., Brunet, R., Coutarel, F., \& Roquelaure, Y. (2017b). Renouvellement des questions et des outils d'évaluation du risque en prévention des TMS à la lumière d'un concept en devenir : la marge de manœuvre. $52^{e}$ Congrès de la Société d'Ergonomie de Langue Française, pp. 473-481.

Noulin, N. (2002). Ergonomie. Toulouse : Octarès Editions.

Ouellet, S., \& Vézina, N. (2014). Work training and MSDs prevention : Contribution of ergonomics. International Journal of Industrial Ergonomics, 44(1), 24-31.

Parot-Schinkel, E., Descatha, A., Ha, C., Petit, A., Leclerc, A., \& Roquelaure, Y. (2012). Prevalence of multisite musculoskeletal symptoms: a French cross-sectional working population-based study. BMC musculoskeletal disorders, 13(1).

Punnett, L., Fine, L. J., Keyserling, W. M., Herrin, G. D., \& Chaffin, D. B. (2000). Shoulder disorders and postural stress in automobile assembly work. Scandinavian journal of work, environment \& health, 26(4), 283-291.

Reed, M., \& Flannagan, C. (2000). Anthropometric and postural variability: limitations of the boundary manikin approach. SAE Transactions: Journal of Passenger Cars, Mechanical Systems, technical Paper $N^{\circ}$ 2000-01-3405.

Ringelberg, J. A., \& Koukoulaki, T. (2002). Risk estimation for musculoskeletal disorders in machinery design: Integrating a user perspective. Brussels : European Trade Union Technical Bureau for Health and Safety

Roquelaure, Y., (2015). Musculoskeletal disorders: a major challenge for occupational risk prevention in Europe. Bruxelles : European Trade Union Institute. Policy Brief, 9.

Roquelaure, Y., (2018). Troubles Musculo-Squelettiques, Risques Psychosociaux et Organisation du travail en milieu. Bruxelles : European Trade Union Institute, R142.

Roquelaure, Y., Ha, C., Leclerc, A., Touranchet, A., Sauteron, M., \& Melchior, M. (2006). Epidemiologic surveillance of upper-extremity musculoskeletal disorders in the working population. Arthritis Care \& Research : Official Journal of the American College of Rheumatology, 55(5), 765-778.

Roquelaure, Y., Leclerc, A., Coutarel, F., Brunet, R., Caroly, S., \& François, D. (2012). Comprendre et intervenir : enquêtes épidémiologiques et approches ergonomiques à propos des troubles musculosquelettiques des membres supérieurs. Risques du travail, la santé négociée, 173-187. 
Roquelaure, Y., Petit, A., Meyer, J. P., Leclerc, A., \& Mairiaux, P. (2013). Surveillance médicoprofessionnelle du risque lombaire pour les travailleurs exposés à des manipulations de charges. Société Française de Médecine du Travail.

Sauter, S-L., \& Swanson, N-G. (1996). An ecological model of musculoskeletal disorders in office work. Psychosocial Factors and Musculoskeletal Disorders, 3(21).

St-Vincent, M., Vézina, N., Bellemare, M., Denis, D., Ledoux, É., \& Imbeau, D. (2014). Ergonomic Intervention. Ed multiMonde: IRSST.

Stock, S. R., Fernandes, R., Delisle, A., \& Vézina, N. (2005). Reproducibility and validity of workers' self-reports of physical work demands. Scandinavian journal of work, environment \& health, 31(6), 409-437.

Takala, E., Pehkonen, I., Forsman, M., Hansson, G., Mathiassen, S. E., Neumann, W., Siogaard, G., Veiersted, R. H., Winkel, J., \& Westgaard, R. (2010). Systematic evaluation of observational methods assessing biomechanical exposures at work. Scandinavian journal of work, environment \& health, 36(3), 24.

Tomás, J.-L., \& Fernandez, G. (2015). Du pouvoir d'agir aux marges de manœuvre : une proposition pour le développement psychologique des gestes. Activités, 12(2), 125.

Vézina, N. (2001). La pratique de l'ergonomie face aux TMS : ouverture à l'interdisciplinarité. Comptes rendus du congrès Société d'Ergonomie de la Langue Francaise - Association Canadienne d'Ergonomie (SELF-ACE), les transformations du travail : enjeux pour l'ergonomie (pp. 44-60), Montréal, Québec.

Westgaard, R. H., \& Winkel, J. (2011). Occupational musculoskeletal and mental health: Significance of rationalization and opportunities to create sustainable production systems - A systematic review. Applied Ergonomics, 42(2), 261-296.

Widanarko, B., Legg, S., Devereux, J., \& Stevenson, M. (2014). The combined effect of physical, psychosocial/organisational and/or environmental risk factors on the presence of work-related musculoskeletal symptoms and its consequences. Applied Ergonomics, 45(6), 1610-1621.

Zare, M. (2015). Représentation partagée des facteurs de risque des troubles musculosquelettiques et comparaison des méthodes d'évaluation : une étude expérimentale dans le secteur de l'assemblage de camions Thèse en ergonomie, Université d'Angers.

Zare, M., Biau, S., Brunet, R., Gourlay, A., \& Roquelaure, Y. (2015). A comparison of neck bending and flexion measurement methods for assessment of ergonomic risk. International journal of occupational safety and ergonomics, 21(3), 330-335.

Zare, M., Biau, S., Brunet, R., \& Roquelaure, Y. (2017). Comparison of three methods for evaluation of work postures in a truck assembly plant. Ergonomics, 60(11), 1551-1563.

Zare, M., Biau, S., Croq, M., \& Roquelaure, Y. (2014). Development of a biomechanical method for ergonomic evaluation: comparison with observational methods. World Academy of Science, Engineering and Technology, International Journal of Social, Behavioral, Educational, Economic, Business and Industrial Engineering, 8(1), 218-222.

\section{RÉSUMÉS}

Les outils d'évaluation du risque Trouble Musculo-Squelettique (TMS) actuellement utilisés dans les industries pour identifier les situations critiques présentent des biais liés à la variabilité des résultats. La validité et la fiabilité du ciblage réalisé avec ces «outils simples » sont remises en 
question ce qui peut représenter un biais possible à l'orientation donnée à l'ensemble de la démarche de prévention des TMS. Dans cette étude, nous proposons d'explorer certains indicateurs déjà publiés sur les notions de régulation et de marge de manœuvre situationnelle (MMS). Ainsi sur un échantillon de situations de travail sélectionné dans une industrie de la métallurgie, nous avons comparé les indicateurs de MMS avec deux méthodes semi-quantitatives et qualitatives pour statuer sur l'état de suffisance de MMS pour chaque situation de travail (suffisance, soupçon d'insuffisance ou insuffisance). Ces deux méthodes montrent des écarts de jugement. Toutefois, elles apportent des informations complémentaires aux outils simples d'évaluation des risques de TMS. Nous proposons des pistes d'amélioration de la méthode semiquantitative pour cibler les situations critiques dès la phase d'évaluation du risque TMS.

The Musculoskeletal Disorder (MSD) risk assessment tools currently used in industries to identify critical situations have shown biases related to the variability of results. The validity and reliability of targeting risk situations with these simple tools are questionable, which might bias the orientation given to the prevention of MSD. This study explores known indicators regarding MSD risk assessment together with notions of regulation and situational operational leeway (SOL). In a sample of selected work situations in the metallurgy industry, we therefore compared the SOL indicators using two semi-quantitative and qualitative methods to determine the level of SOL sufficiency for each situation. The results of these two methods show differences in judgment. These methods nevertheless provide additional information that complement simple MSD risk assessment tools. We propose ways to improve semi-quantitative methods to target critical situations as from the MSD risk assessment phase.

\section{INDEX}

Keywords : practitioners, musculoskeletal disorders, critical situations, situational operational leeway, semi-quantitative methods

Mots-clés : préventeurs, troubles musculo-squelettiques, situations critiques, marge de manœuvre situationnelle, méthode semi-quantitative

\section{AUTEURS}

\section{MAXIME NORVAL}

INSERM U1085, Équipe Ester, Université Bretagne - Pays de la Loire, UFR Santé - Faculté de médecine, 28 Rue Roger Amsler, F-49045 Angers Cedex 1, France, maximenorval@hotmail.com

\section{MOHSEN ZARE}

ELLIAD EA4661, Pôle ERCOS, UTBM-Université Bourgogne Franche-Comté, Cours Louis LeprinceRinguet, 25200 Montbéliard, France

\section{RENÉ BRUNET}

IRSET-INSERM UMR_S 1 085, Équipe Ester, Université Angers Bretagne Loire, 28 rue Amsler 49045 Angers, France

\section{FABIEN COUTAREL}

ACTÉ EA4281, Université Clermont-Auvergne, UFR STAPS, Campus Universitaire des Cézeaux, 3 rue de la Chebarde, 63178 Aubiere, France 


\section{YVES ROQUELAURE}

IRSET-INSERM UMR_S 1 085, Équipe Ester, Université Angers Bretagne Loire, 28 rue Amsler 49045 Angers, France 